\title{
Cores of Games With Restricted Cooperation
}

\author{
By U. Faigle ${ }^{1}$
}

Abstract: Games with restricted cooperation are cooperative $N$-person games with sidepayments, where the collection of feasible coalitions need not comprise all subsets of players and thus is restricted. We study balanced and completely balanced games in this context and derive the corresponding core theorems from a sandwich theorem for set functions within the setting of linear programming. In particular, we discuss general convex games, which Edmonds and Giles (1977) have shown to be of particular importance also in combinatorial optimization.

Zusammenfassung: Spiele mit beschränkter Kooperation sind kooperative $N$-Personenspiele mit Nebenzahlungen, wobei nicht jede Teilmenge von Spielern zulässig zu sein braucht. In diesem Sinn sind die Kooperationsmöglichkeiten beschränkt. Balancierte und vollständig balancierte Spiele werden in diesem Zusammenhang untersucht. Die entsprechenden Sätze über đie Existenz von Kernen werden von einem Sandwichsatz über Mengenfunktionen im Rahmen der linearen Programmierung abgeleitet. Insbesondere werden allgemeine konvexe Spiele diskutiert, deren Bedeutung auch für die kombinatorische Optimierung Edmonds and Giles (1977) aufgezeigt haben.

Key words: games, restricted cooperation, core, convex functions.

\section{Introduction}

The model commonly employed for the analysis of cooperative $N$-person games with sidepayments places several structural restrictions on the collection of feasible coalitions. It is assumed that this collection forms an algebra, i.e., that unions and complements of feasible coalitions again are feasible coalitions of players. If all individual players are allowed to form coalitions by themselves, then the standard model considers every subset of players as a feasible coalition.

In many important situations, however, this model does not apply. For example, a player may have agreed in a treaty to enter a coalition as soon as some other player enters it. The latter player will, therefore, be unable to form a coalition by himself

1 Ulrich Faigle, University of Twente, Faculty of Applied Mathematics, P.O. Box 217, 7500 AE Enschede, The Netherlands. 
even if the first player should be able to do so. Hence it appears necessary to investigate a more general model for cooperative games in which cooperation among players is restricted to some prescribed collection of subsets, the feasible coalitions of the game, without any à priori structure. The difficulty, of course, arises with the question about the appropriate solution concepts of such games with restricted cooperation.

In this paper, we study the core of general cooperative games. As in the classical case, every vector in the core of a game is a solution to the problem of finding a modular function that dominates the value function of the game on the feasible coalitions and has a prescribed value for the total set of players. Thus we may derive necessary and sufficient conditions for the existence of the core from a general sandwich theorem for set functions in Section 2, which can be interpreted as a statement about certain associated primal and dual linear programs - an idea that goes back to Bondareva (1963). In particular, also the notion of balanced games is meaningful in this context and we state the corresponding core theorems in Section 3. Moreover, we give conditions for a game to be completely balanced, which means that for every individual player a non-negative return can be guaranteed.

In Section 4 we look at general convex games. They provide a very general model for game theoretic convexity and, in fact, are a link to combinatorial optimization, where Edmonds and Giles (1977) have shown such structures to play an important role. Contrary to classical convex games investigated by Shapley (1971), general convex games need not have a non-empty core. Obviously necessary conditions, however, turn out to be also sufficient for the existence of a core. Moreover, the positive core of a completely balanced convex game always arises as the core of some convex game in which all subsets of players are feasible coalitions.

\section{A Sandwich Theorem}

Let $S$ be a finite set and $F$ a non-empty collection of feasible subsets of $S$. At the outset, $\emptyset \in F$ is not assumed and we write

$$
F_{0}=F \cup\{\emptyset\}
$$

We furthermore assume to be given two arbitrary functions $f: F \rightarrow \mathbb{R}$ and $g: F \rightarrow \mathbb{R}$ such that

$$
\begin{array}{ll}
g(A) \leqslant f(A) & \text { for all } A \in F \\
g(\emptyset)=f(\emptyset)=0 & \text { if } \emptyset \in F .
\end{array}
$$


Denoting by $\mathbb{R}^{S}$ the $|S|$-dimensional Euclidean space of all vectors whose components are indexed by elements of $S$, each $x \in \mathbb{R}^{S}$ gives rise to a function $x: F \rightarrow \mathbb{R}$ via

$$
x(A)=\sum_{s \in A} x_{s} \quad \text { for all } A \in F
$$

For the formulation of our main result, we need the indicator function $1_{U}: S \rightarrow \mathbb{R}$ for the subset $U \subseteq S$ given by

$$
1_{U}(s)= \begin{cases}1 & \text { if } s \in U \\ 0 & \text { otherwise. }\end{cases}
$$

Theorem 1: Under the above hypotheses,

a) The following are equivalent:

$\left.a_{1}\right)$ There exists some $x \in \mathbb{R}^{S}$ such that

$g(A) \leqslant x(A) \leqslant f(A)$ for all $A \in F$.

a ) For all $A_{1}, \ldots, A_{n}, B_{1}, \ldots, B_{m} \in F$ with $\sum_{i=1}^{n} 1_{A_{i}}=\sum_{j=1}^{m} 1_{B_{j}}$,

$$
\sum_{i=1}^{n} g\left(A_{i}\right) \leqslant \sum_{j=1}^{m} f\left(B_{j}\right)
$$

b) The following are equivalent:

$b_{1}$ ) There exists some $x \in \mathbb{R}^{S}, x \geqslant 0$, such that

$g(A) \leqslant x(A) \leqslant f(A)$ for all $A \in F$.

$\mathrm{b}_{2}$ ) For all $A_{1}, \ldots, A_{n}, B_{1}, \ldots, B_{m} \in F_{0}$ with $\sum_{i=1}^{n} 1_{A} \leqslant \sum_{j=1}^{m} 1_{B_{j}}$,

$$
\sum_{i=1}^{n} g\left(A_{i}\right) \leqslant \sum_{j=1}^{m} f\left(B_{j}\right)
$$


Remark: Kindler (1986) has derived an analogue of Theorem 1 for possibly infinite $S$ from Kaufman's (1966) theorem on additive functionals on semigroups provided $F$ is closed union and intersection with $\emptyset \in F$ and $f$ submodular, i.e.,

$$
f(A \cup B)+f(A \cap B) \leqslant f(A)+f(B) .
$$

Proof of Theorem 1: To verify part a), observe first that $\left(a_{2}\right)$ is necessary for $\left(a_{1}\right)$ to hold. To see also the sufficiency of $\left(a_{2}\right)$, note that $\left(a_{1}\right)$ amounts to saying that the primal linear program

$$
\max 0 \cdot x
$$

such that

$$
\begin{aligned}
& x(A) \leqslant f(A) \quad \text { for all } A \in F \\
& -x(A) \leqslant-g(A) \quad \text { for all } A \in F \\
& x \in \mathbb{R}^{S} \quad \text { unrestricted }
\end{aligned}
$$

has a (feasible) solution. According to the duality theorem of linear programming (see, e.g., Chvátal 1983), this is the case exactly when the associated dual linear program

$$
\min \sum_{A \in F} f(A) \cdot y_{A}-\sum_{A \in F} g(A) z_{A}
$$

such that

$$
\begin{array}{ll}
\sum_{\substack{A \in F \\
s \in A}} y_{A}=\sum_{\substack{A \in F \\
s \in A}} z_{A} & \text { for all } s \in S \\
y_{A} \geqslant 0, z_{A} \geqslant 0 & \text { for all } A \in F
\end{array}
$$

does not admit of a (feasible) solution yielding a negative value of the dual objective function. We will show that the latter property holds. 
Suppose there are non-negative $|\boldsymbol{F}|$-dimensional vectors y and $z$ such that for all $s \in S$,

$$
\sum_{\substack{A \in F \\ s \in A}} y_{A}=\sum_{\substack{A \in F \\ s \in A}} z_{A}
$$

and

$$
\sum_{A \in F} f(A) y_{A}<\sum_{A \in F} g(A) z_{A}
$$

There is no loss in generality if we assume that $y$ and $z$ have rational components. We now clear the denominators and thus see that $y$ and $z$ may be chosen to have integer components only. But then $y$ and $z$ obviously violate $\left(a_{2}\right)$.

Part b) follows exactly the same way upon taking into account that the primal linear program stipulates $x \geqslant 0$ and, therefore, the equality constraints in the dual program have to be modified to inequalities

$$
\sum_{\substack{A \in F \\ s \in A}} y_{A} \geqslant \sum_{\substack{A \in F \\ s \in A}} z_{A} \quad \text { for all } s \in S
$$

A specialization of Theorem 1 will be useful in the analysis of the existence of the core of a game in the next section. To this end, we assume that there exists a subsystem $L \subseteq F$ of subsets of $S$ satisfying the following conditions:

(i) $S \in L$

(ii) For all $A, B \in L$ such that there exists some $F \in F$ with $F \subseteq A \cap B$, we have $A \cap B \in L$.

Hence we may associate with each $F \in F$ a unique set $\bar{F} \in L$ via

$$
\bar{F}=\cap\{L \in L: F \subseteq L\}
$$


Corollary 2: The following are equivalent:

$\left(c_{1}\right)$ There exists some $x \in \mathbb{R}^{S}, x \geqslant 0$, such that

$$
\begin{aligned}
& g(A) \leqslant x(A) \quad \text { for all } A \in F \\
& g(L)=x(L) \quad \text { for all } L \in L .
\end{aligned}
$$

$\left(c_{2}\right)$ For all $A_{1}, \ldots, A_{n} \in F_{0}, L_{1}, \ldots, L_{m} \in L$ with $\sum_{i=1}^{n} 1_{A_{i}} \leqslant \sum_{j=1}^{m} 1_{L_{i}}$,

$$
\sum_{i=1}^{n} g\left(A_{i}\right) \leqslant \sum_{j=1}^{m} g\left(L_{j}\right)
$$

Proof: Choosing $f=x$ for an application of Theorem 1, it is immediate that $\left(c_{1}\right)$ implies $\left(\mathrm{c}_{2}\right)$.

Conversely, if ( $\left.\mathrm{c}_{2}\right)$ holds, we define for all $A \in F$,

$$
f(A)=g(\bar{A}) .
$$

In view of $A \subseteq \bar{A},\left(\mathrm{c}_{2}\right)$ guarantees $g(A) \leqslant f(A)$.
Furthermore, for all $A_{1}, \ldots, A_{n}, B_{1}, \ldots, B_{m} \in F_{0}$ with $\sum_{i=1}^{n} 1_{A_{i}} \leqslant \sum_{j=1}^{m} 1_{B_{j}}$, we have $\sum_{i=1}^{n} 1_{A_{i}} \leqslant \sum_{j=1}^{m} 1_{\bar{B}_{j}}$ and therefore

$$
\sum_{i=1}^{n} g\left(A_{i}\right) \leqslant \sum_{j=1}^{m} g\left(\bar{B}_{j}\right)=\sum_{j=1}^{m} f\left(B_{j}\right)
$$

Thus condition $\left(\mathrm{b}_{2}\right)$ of Theorem 1 is satisfied. Because $g(L)=f(L)$ for every $L \in L$, also $\left(c_{1}\right)$ must be true. 
If we do not insist that the solution vector $x \in \mathbb{R}^{S}$ be non-negative, we obtain

Corollary 3: The following are equivalent:

$\left(d_{1}\right)$ There exists some $x \in \mathbb{R}^{S}$ such that

$$
\begin{aligned}
& g(A) \leqslant x(A) \quad \text { for all } A \in F \\
& g(L)=x(L) \quad \text { for all } L \in L .
\end{aligned}
$$

$\left(\mathrm{d}_{2}\right)$ For all $A_{1}, \ldots, A_{n} \in F, L_{1}, \ldots, L_{m} \in L$ with $\sum_{i=1}^{n} 1_{A_{i}}=\sum_{j=1}^{m} 1_{L_{j}}$,

$$
\sum_{i=1}^{n} g\left(A_{i}\right) \leqslant \sum_{j=1}^{m} g\left(L_{j}\right)
$$

Proof: Choose a vector $c \in \mathbb{R}^{S}$ with the property

$$
g\left(A_{1}\right)+c\left(A_{1}\right) \leqslant g\left(A_{2}\right)+c\left(A_{2}\right) \text { for all } A_{1} \subseteq A_{2} \in F \text { : }
$$

Setting $f(A)=g(\bar{A})+c(\bar{A})$, we conclude as before from Theorem 1 the equivalence of $\left(\mathrm{d}_{1}^{\prime}\right)$ There exists some $x \in \mathbb{R}^{S}$ such that

$$
\begin{array}{ll}
g(A)+c(A) \leqslant x(A)+c(A) & \text { for all } A \in F \\
g(L)+c(L)=x(L)+c(L) & \text { for all } L \in L .
\end{array}
$$

$\left(\mathrm{d}_{2}^{\prime}\right)$ For all $A_{1}, \ldots, A_{n} \in F, L_{1}, \ldots, L_{m} \in L$ with $\sum_{i=1}^{n} 1_{A_{i}}=\sum_{j=1}^{m} 1_{L_{j}}$,

$$
\sum_{i=1}^{n}\left(g\left(A_{i}\right)+c\left(A_{i}\right)\right) \leqslant \sum_{i=1}^{m}\left(g\left(L_{j}\right)+c\left(L_{j}\right)\right)
$$

These conditions, however, are equivalent to $\left(d_{1}\right)$ and $\left(d_{2}\right)$. 
We remark that in the conditions $\left(b_{2}\right)$ and $\left(c_{2}\right)$ we may, of course, replace $F_{0}$ by $F$ if we state that $f$ be non-negative on $F$ or $L$.

\section{Games With Restricted Cooperation}

We propose a general model for a cooperative game with a finite set $S$ of players. It does not assume that every coalition $U \subseteq S$ of players be feasible and thus takes into account the situation, for instance, where some players may only join a coalition if some other players join the same coalition as well.

Definition: A (finite) game with restricted cooperation is a quadruple $\Gamma=\left(S, F, v, v_{0}\right)$, where $S$ is the finite set of players, $F$ a nonempty collection of subsets of $S$ called feasible coalitions, $v: F \rightarrow \mathbb{R}$ the value function, with $v(\phi)=0$ and $v_{0} \in \mathbb{R}$ the value of the game $\Gamma$. If $v$ and $v_{0}$ are non-negative, $\Gamma$ is a positive game.

A solution of the game $\Gamma$ is a fair distribution of its value $v_{0}$ among the players. As usual, we therefore define the core $C(v)$ of the game $\Gamma$ to consist of all undominated imputations, i.e., vectors $x \in \mathbb{R}^{S}$ such that

(i) $\sum_{s \in A} x_{s} \geqslant v(A)$ for all $A \in F$

(ii) $\sum_{s \in S} x_{s}=v_{0}$.

Note that we do not require that an imputation necessarily be non-negative. Indeed, individual players may end up with a negative payoff if they do not have the strength to secure a non-negative pay off by forming individual coalitions. As far as the existence of $C(v)$ is concerned, it is clear that our model remains essentially the same if we assume $S \in F$ and $v(S)=v_{0}$. Hence we will make this assumption for the following discussion. Our aim is to show that the classical theorems on the existence of cores also hold true in this wider context.

Call the game $\Gamma=\left(S, F, v, v_{0}\right)$ balanced if for all $A_{1}, \ldots, A_{n} \in F$ and $m \in \mathbb{N}$ :

$$
\frac{1}{m} \sum_{i=1}^{n} 1_{A_{i}}=1_{S} \quad \text { implies } \quad \frac{1}{m} \sum_{i=1}^{n} v\left(A_{i}\right) \leqslant v_{0} .
$$


Then we obtain a generalization of the theorem of Bondareva (1963) (see also Shapley 1967 ) by taking $L=\{S\}$ in Corollary 3 .

Theorem 4: The game $\Gamma=\left(S, F, v, v_{0}\right)$ is balanced if and only if $C(v) \neq \emptyset$.

The game $\Gamma=\left(S, F, v, v_{0}\right)$ is said to be exact if for all $A \in F$,

$$
v(A)=\min _{x \in C(v)} x(A)
$$

Choosing $L=\{A, S\}$ in Corollary 3 , we thus arrive at the analogue of a result due to Schmeidler (1972):

Theorem 5: $\Gamma=\left(S, F, v, v_{0}\right)$ is exact if and only if for all $A, A_{1}, \ldots, A_{n} \in F$ and $m, k \in \mathbb{N}$,

$$
\sum_{i=1}^{n} 1_{A_{i}}=m \cdot 1_{S}+k \cdot 1_{A} \quad \text { implies } \quad \sum_{i=1}^{n} v\left(A_{i}\right) \leqslant m v_{0}+k v(A) .
$$

Let us define the positive core of the game $\Gamma=\left(S, F, v, v_{0}\right)$ as

$$
C^{+}(v)=\{x \in C(v): x \geqslant 0\}
$$

Then even a positive balanced game may have $C^{+}(v)=\emptyset$. To see this, take $F=\{A, S\}$ with $v(A)>v_{0}>0$. Hence calling $\Gamma$ completely balanced if $C^{+}(v) \neq \emptyset$, Corollary 2 yields

Theorem 6: $\Gamma=\left(S, F, v, v_{0}\right)$ is completely balanced if and only if for all $A_{1}, \ldots, A_{n} \in F_{0}$ and $m \in \mathbb{N}$,

$$
\frac{1}{m} \sum_{i=1}^{n} 1_{A_{i}} \leqslant 1_{S} \quad \text { implies } \quad \frac{1}{m} \sum_{i=1}^{n} v\left(A_{i}\right) \leqslant v_{0} .
$$

Although a positive balanced game need not be completely balanced, certain properties of the collection $\boldsymbol{F}$ of feasible coalitions may ensure that a game is completely balanced if it is balanced at all. From Theorem 4 and Theorem 6, for example, it is not hard to derive the following sufficient condition. 
Proposition 7: Let $\Gamma=\left(S, F, v, v_{0}\right)$ be a positive balanced game such that for all $A, B \in F, A \cap B \in F$ and $S-A \in F$ holds. Then $\Gamma$ is completely balanced.

It is also apparent that $C(v)=C^{+}(v)$ if each player of the game $\Gamma$ can form a feasible coalition without including any other player. The converse of the statement is not necessarily true as the next example demonstrates.

Example 8: Let $S=\{1,2,3\}$ and let $F$ consist of $S$ together with all 2-element subsets of $S$. If

$$
v(A)= \begin{cases}3 & \text { if } A=S \\ 2 & \text { otherwise }\end{cases}
$$

then $C(v)=C^{+}(v)=\{(1,1,1)\} \neq \emptyset$

Nevertheless, for a large class of games, a certain converse holds.

Theorem 9: Let $\Gamma=\left(S, F, v, v_{0}\right)$ be a positive balanced game such that $A \cap B \in F$ whenever $A, B \in F$. Then $C(v)=C^{+}(v)$ if and only if $\{s\} \in F$ for all $s \in S$.

Proof: Suppose there exists $a \in S$ with $\{a\} \notin F$.

We claim that $C(v) \neq C^{+}(v)$.

Let $A$ be the smallest feasible coalition containing $a$. Since $A \neq\{a\}$, there exists some $b \in A-\{a\}$. Consider any imputation $x \in C(v)$ together with an arbitrary real number $\epsilon>0$ and define $x^{\epsilon} \in \mathbb{R}^{S}$ via

$$
x_{s}^{\epsilon}= \begin{cases}x_{s}-\epsilon & \text { if } s=a \\ x_{s}+\epsilon & \text { if } s=b \\ x_{s} & \text { otherwise. }\end{cases}
$$

Then $x^{\epsilon} \in C(v)$ and $x^{\epsilon} \notin C^{+}(v)$ if $\epsilon$ is large enough. 
Note that the proof of Theorem 9 moreover shows: if the feasible coalitions of the positive balanced game $\Gamma$ are closed under intersection, then $C(v)$ is unbounded unless $C(v)=C^{+}(v)$. In other words, even membership in a strong coalition is no guarantee for a non-negative individual payoff if one lacks the strength to form an individual coalition.

\section{Convex Games}

Recall that a set function $f$ is convex (a.k.a. supermodular) if it satisfies the inequality

$$
f(A)+f(B) \leqslant f(A \cup B)+f(A \cap B)
$$

for arbitrary subsets $A$ and $B$. It is well-known that every game with a convex value function is balanced if every subset of players forms a feasible coalition (see, e.g., Shapley 1971). We will now discuss convexity in the context of games with restricted cooperation. This broader concept of convexity corresponds to the analogous extension of the notion of submodularity in combinatorial optimization, where it has proved very useful (see, e.g. Edmonds and Giles 1977 and Fujishige 1984).

Consider the game $\Gamma=\left(S, F, v, v_{0}\right)$, where we again assume $S \in F$ and $v(S)=v_{0}$. Let $\bar{F}$ consist of all those subsets $A \subseteq S$ which can be written as

$$
A=A_{1} \cup A_{2} \cup \ldots \cup A_{k},
$$

where $A_{1}, A_{2}, \ldots, A_{k}$ are pairwise disjoint feasible coalitions of $\Gamma$. We define the set function $\bar{v}$ on $\overline{\boldsymbol{F}}$ via

$$
\bar{v}(A)=\max \sum_{i} v\left(A_{i}\right)
$$

where the maximum is taken over all representations of $A$ as a union of pairwise disjoint feasible coalitions. Setting $\bar{v}_{0}=\bar{v}(S)$, we thus obtain a game $\bar{\Gamma}=\left(S, \bar{F}, \bar{v}, \bar{v}_{0}\right)$ and observe 
Lemma 10: $\vec{F}$ is closed under taking disjoint unions of coalitions and $\vec{v}$ is superadditive, i.e., for all $A, B \in \vec{F}$ with $A \cap B=\emptyset$,

$$
\bar{v}(A)+\bar{v}(B) \leqslant \bar{v}(A \cup B)
$$

Moreover, if $\bar{v}_{0}=v_{0}$, then $C(\bar{v})=C(v)$.

Following Edmonds and Giles (1977) we say that two subsets $A, B \subseteq S$ form a crossing pair if $A \cup B \neq S$ and $A \cap B \neq \emptyset$ and, furthermore, $A \backslash B \neq \emptyset \neq B \backslash A$. $F$ is a crossing family if $A \cup B \in F$ and $A \cap B \in F$ whenever $A, B \in F$ are a crossing pair.

We now say that the game $\Gamma=\left(S, F, v, v_{0}\right)$ is convex if $F$ is a crossing family and for every crossing pair $A, B \in F$

$$
v(A)+v(B) \leqslant v(A \cup B)+v(A \cap B) .
$$

A special case is given by an intersecting convex game $\Gamma$, where for all $A, B \in F$ with $A \cap B \neq \emptyset, A \cup B \in F$ and $A \cap B \in F$ and

$$
v(A)+v(B) \leqslant v(A \cup B)+v(A \cap B)
$$

Lemma 11: Let $\Gamma=\left(S, F, v, v_{0}\right)$ be an intersecting convex game. Then $\bar{F}$ is closed under union and intersection of coalitions and for all $A, B \in \bar{F}$

$$
\bar{v}(A)+\bar{v}(B) \leqslant \bar{v}(A \cup B)+\bar{v}(A \cap B) .
$$

Proof: $\emptyset \in \bar{F}$ is obtained as the empty representation of feasible coalitions of $\Gamma$. The closedness under intersection is a direct consequence of de Morgan's laws:

$$
\bigcup_{i=1}^{n} A_{i} \cap \bigcup_{j=1}^{m} B_{j}=\bigcup_{i, j}\left(A_{i} \cap B_{j}\right)
$$

Suppose that $\bar{v}$ is not convex on $\bar{F}$ and that

$$
A=A_{1} \cup \ldots \cup A_{n} \quad \text { with } \quad \sum_{i=1}^{n} v\left(A_{i}\right)=\bar{v}(A)
$$




$$
B=B_{1} \cup \ldots \cup B_{m} \quad \text { with } \sum_{j=1}^{m} v\left(B_{j}\right)=\bar{v}(B)
$$

yield

$$
\bar{v}(A)+\bar{v}(B)>\bar{v}(A \cup B)+\bar{v}(A \cap B)
$$

and that $n$ is as small as possible among all such counterexamples.

$$
\begin{aligned}
& \text { Assume first } n \geqslant 2 \text { and let } A^{\prime}=A_{1} \cup \ldots \cup A_{n-1} . \text { Then } \\
& \bar{v}\left(A^{\prime} \cup\left(A_{n} \cup B\right)\right)+\bar{v}\left(A^{\prime} \cap\left(A_{n} \cup B\right)\right) \geqslant \bar{v}\left(A^{\prime}\right)+\bar{v}\left(A_{n} \cup B\right) \\
& \bar{v}\left(A_{n} \cup B\right)+\bar{v}\left(A_{n} \cap B\right) \geqslant \bar{v}\left(A_{n}\right)+\bar{v}(B)
\end{aligned}
$$

Since $A_{n} \cap B$ and $A^{\prime} \cap\left(A_{n} \cup B\right)$ are disjoint, we have

$$
\bar{v}(A \cap B) \geqslant \bar{v}\left(A_{n} \cap B\right)+\bar{v}\left(A^{\prime} \cap\left(A_{n} \cup B\right)\right) .
$$

Therefore, $\bar{v}(A)=\bar{v}\left(A^{\prime}\right)+\bar{v}\left(A_{n}\right)$ yields

$$
\bar{v}(A)+\bar{v}(B) \leqslant \bar{v}(A \cup B)+\bar{v}(A \cap B),
$$

a contradiction. Hence $n=1$ and we may assume as well that also $m$ has been chosen as small as possible. Then $A=A_{1}$ must have a non-empty intersection with each of the $B_{j}$ 's, and thus also the sets $A_{1} \cup B_{1}, A_{1} \cup B_{1} \cup B_{2}, \ldots$ are members of the intersecting family $F$. Now

$$
\begin{aligned}
\bar{v}(A)+\bar{v}(B) & =v\left(A_{1}\right)+v\left(B_{1}\right)+\ldots+v\left(B_{m}\right) \\
& \leqslant v\left(A_{1} \cup B_{1}\right)+v\left(B_{2}\right)+\ldots+v\left(B_{m}\right)+v\left(A_{1} \cap B_{1}\right) \\
& \leqslant v\left(A_{1} \cup B_{1} \cup B_{2}\right)+v\left(B_{3}\right)+\ldots+v\left(B_{m}\right)+v\left(A_{1} \cap B_{1}\right)+v\left(A_{1} \cap B_{2}\right) \\
& \vdots \\
& \leqslant v(A \cup B)+v\left(A_{1} \cap B_{1}\right)+\ldots+v\left(A_{1} \cap B_{m}\right) \\
& \leqslant \bar{v}(A \cup B)+\bar{v}(A \cap B),
\end{aligned}
$$

a contradiction, which completes the proof. 
Proposition 12: Let $\Gamma=\left(S, F, v, v_{0}\right)$ be an intersecting convex game. Then $\Gamma$ is balanced if and only if for all pairwise disjoint feasible coalitions $A_{1}, A_{2}, \ldots, A_{n} \in F$ with $A_{1} \cup A_{2} \cup \ldots \cup A_{n}=S$,

$$
v\left(A_{1}\right)+v\left(A_{2}\right)+\ldots+v\left(A_{n}\right) \leqslant v_{0} .
$$

Moreover, $\Gamma$ is completely balanced if and only if for all pairwise disjoint feasible coalitions $A_{1}, A_{2}, \ldots, A_{n} \in F_{0}$,

$$
v\left(A_{1}\right)+v\left(A_{2}\right)+\ldots+v\left(A_{n}\right) \leqslant v_{0} .
$$

Proof: The conditions are clearly necessary and ensure $v_{0}=\bar{v}_{0}$. To see that they are also sufficient, we consider the associated convex game $\vec{\Gamma}$.

Choose a vector $c \in \mathbb{R}^{S}$ such that for all $A \subseteq B \in \bar{F}$,

$$
\bar{v}(A)+c(A) \leqslant \bar{v}(B)+c(B)
$$

and define for every subset $X \subseteq S$,

$$
f(X)=\max \{\bar{v}(A)+c(A): A \subseteq X, A \in \bar{F}\}
$$

Since $\bar{v}+c$ is convex and monotone on $\overline{\boldsymbol{F}}, f$ is convex and monotone with respect to all subsets of $S$ and therefore defines a convex game in the sense of Shapley (1971) with value $f(S)=v_{0}+c(S)$. Hence $C(f) \neq \emptyset$ and the observation

$$
x-c \in C(\bar{v}) \text { if } x \in C(f)
$$

implies $C(\bar{v})=C(v) \neq \emptyset$.

For the second statement we define for $X \subseteq S$,

$$
f^{\prime}(X)=\max \{\bar{v}(A): A \subseteq X, A \in \bar{F}\} .
$$


Again $f^{\prime}$ is convex and monotone. Furthermore, the condition given guarantees $f^{\prime}(S)=$ $\bar{v}(S)=v_{0}$. Now $C^{+}\left(f^{\prime}\right) \neq \emptyset$ and

$x \in C^{+}(\bar{v})$ if and only if $x \in C^{+}\left(f^{\prime}\right)$

which finishes the proof.

Note that the proof of Proposition 12 exhibits an intersecting convex game $\Gamma$ to be completely balanced if and only if its non-negative core $C^{+}(v)$ is the core of some positive convex game in which all subsets of players constitute feasible coalitions.

We now discuss the case where the convex game $\Gamma=\left(S, F, v, v_{0}\right)$ is not necessarily intersecting. Then the previous construction may not work as $F$ need not be closed under union and intersection (to see this, take, for example, $S=\{1,2,3\}$ and $F=$ $\{\{1,2\},\{1,3\},\{2,3\}\})$. Note, however, that the set family dual to $F$ is intersecting. Therefore, we consider the collection

$$
D=\{D \subseteq S: S \backslash D \in \bar{F}\}
$$

of complements of members of $\bar{F}$. As in Lemma 11, we have for all $A, B \in \bar{F}$ and $A \cup B \neq S$ if $A \cap B \neq \emptyset$,

$$
\bar{v}(A)+\bar{v}(B) \leqslant \bar{v}(A \cup B)+\bar{v}(A \cap B)
$$

Hence we obtain for all $D, E \in D$ with $D \cap E \neq \emptyset$,

$$
w(D)+w(E) \geqslant w(D \cup E)+w(D \cap E)
$$

where $w(D)=v_{0}-\bar{v}(S \backslash D)$ is submodular. Consider the collection $\bar{D}$ of all subsets of $S$ which can be written as disjoint unions of members of $D$. As before, we then conclude that $\bar{D}$ is closed under union and intersection and for all $D, E \in \bar{D}$,

$$
\bar{w}(D)+\bar{w}(E) \geqslant \bar{w}(D \cup E)+\bar{w}(D \cap E),
$$


where

$$
\bar{w}(D)=\min \sum_{i=1}^{n} w\left(D_{i}\right)
$$

with the minimum taken over all representations $D=D_{1} \cup \ldots \cup D_{n}$ by pairwise disjoint members of $\boldsymbol{D}$. Hence we obtain

$$
F^{*}=\{A \subseteq S: S \backslash A \in \bar{D}\}
$$

as a collection of subsets of $S$ that contains $F$ and is closed under union and intersection. Moreover, for all $A, B \in F^{*}$,

$$
v^{*}(A)+v^{*}(B) \leqslant v^{*}(A \cup B)+v^{*}(A \cap B),
$$

where $v^{*}(A)=\bar{w}(S)-\bar{w}(S \backslash A)$. Thus $\Gamma^{*}=\left(S, F^{*}, v^{*}, v^{*}(S)\right)$ is a convex game with $C\left(v^{*}\right) \neq \phi$.

Because $v^{*}(S)=\bar{w}(S)$, it is clear that $v^{*}(S) \leqslant v_{0}$. On the other hand, tracing back the construction for $v^{*}$, one quickly verifies for each $x \in \mathbb{R}^{S}$ with $x(S)=v_{0}$,

$$
x \in C(v) \text { implies } x(S) \leqslant \bar{w}(S) .
$$

Hence $v^{*}(S)=v_{0}$ is necessary for $C(v) \neq \phi$. Conversely, this condition is also sufficient since $C\left(v^{*}\right) \neq \phi$. Hence we obtain the following result.

Theorem 13: Let $\Gamma=\left(S, F, v, v_{0}\right)$ be any convex game. Then $\Gamma$ is balanced if and only if

$$
v_{0}=v^{*}(S)
$$

Moreover, $\Gamma$ is completely balanced if and only if

$$
v_{0}=v^{*}(S) \geqslant 0
$$


One can also characterize general balanced convex games in the spirit of Proposition 12 by employing the technique of "uncrossing dual variables" introduced by Edmonds and Giles (1977). We say that a collection $A_{1}, \ldots, A_{n}$ of (not necessarily distinct) members of $F$ is cross-free if there are no two members $A_{i}, A_{j}$ such that $A_{i} \nsubseteq A_{j} \nsubseteq A_{i}$, $A_{i} \cap A_{j} \neq \phi$, and $A_{i} \cup A_{j} \neq S$.

Proposition 14: Let $\Gamma=\left(S, F, v, v_{0}\right)$ be any convex game. Then $\Gamma$ is balanced if and only if for every cross-free $A_{1}, \ldots, A_{n} \in F, m \in \mathbb{N}$,

$$
\frac{1}{m} \sum_{i=1}^{n} 1_{A_{i}}=1_{S} \text { implies } \frac{1}{m} \sum_{i=1}^{n} v\left(A_{i}\right) \leqslant v_{0} \text {. }
$$

Moreover, $\Gamma$ is completely balanced if and only if for every crossfree $A_{1}, \ldots, A_{n} \in F_{0}$, $m \in \mathbb{N}$,

$$
\frac{1}{m} \sum_{i=1}^{n} 1_{A_{i}}=1_{S} \quad \text { implies } \quad \frac{1}{m} \sum_{i=1}^{n} v\left(A_{i}\right) \leqslant v_{0} .
$$

Proof: We prove the first statement and note that for $\Gamma$ to be balanced the stated con. dition must be satisfied.

To show that the condition is also sufficient, assume that $\Gamma$ is not balanced. Then there is a collection $A_{1}, \ldots, A_{n} \in F$ and an integer $m \in \mathbb{N}$ such that

$$
\frac{1}{m} \sum_{i=1}^{n} 1_{A_{i}}=1_{S} \text { but } \frac{1}{m} \sum_{i=1}^{n} v\left(A_{i}\right)>v_{0} .
$$

We must show that this collection can be assumed to be cross-free. To see this, choose among all these collections one that minimizes

$$
\sum_{i=1}^{n}\left|A_{i}\right| \cdot\left|S \backslash A_{i}\right|
$$

We claim that this collection is cross-free. Suppose to the contrary that $A_{1} \cap A_{2} \neq \phi$, $A_{1} \cup A_{2} \neq S, A_{1} \nsubseteq A_{2} \nsubseteq A_{1}$, say. Then consider $A_{1}^{\prime}, A_{2}^{\prime}, \ldots, A_{n}^{\prime} \in F$, where 


$$
\begin{aligned}
& A_{1}^{\prime}=A_{1} \cup A_{2} \\
& A_{2}^{\prime}=A_{1} \cap A_{2} \\
& A_{i}^{\prime}=A_{i} \quad i=3,4, \ldots, n .
\end{aligned}
$$

Then clearly

$$
\frac{1}{m} \sum_{i=1}^{n} 1_{A_{i}}=1_{S} \text { and } \frac{1}{m} \sum_{i=1}^{n} v\left(A_{i}^{\prime}\right)>v_{0}
$$

because $v\left(A_{1}\right)+v\left(A_{2}\right) \leqslant v\left(A_{1} \cup A_{2}\right)+v\left(A_{1} \cap A_{2}\right)$. But

$$
\sum_{i=1}^{n}\left|A_{i}^{\prime}\right| \cdot\left|S \backslash A_{i}^{\prime}\right|<\sum_{i=1}^{n}\left|A_{i}\right| \cdot\left|S \backslash A_{i}\right|,
$$

contradicting the choice of $A_{1}, \ldots, A_{n}$.

Acknowledgment: The author thanks P. Schuld, E. Weiss, A. Münstermann and the referees for their careful reading and remarks that helped enhance the presentation.

\section{References}

Bondareva ON (1963) Some applications of linear programming methods to the theory of cooperative games. Problemy Kibernet 10:119-139 (in Russian)

Chvátal V (1983) Linear programming. WH Freeman, New York

Edmonds J, Giles R (1977) A min-max relation for submodular functions on graphs. Annals of Discrete Math 1:185-204

Fujishige S (1984) Submodular systems and related topics. Math Programming Study 22:113-131

Kaufman R (1966) Interpolation of additive functionals. Studia Math 27:269-272

Kindler J (1986) A Mazur-Orlicz type theorem for submodular set functions. Journ Math Analysis and Appl 120:533-546

Schmeidler D (1972) Cores of exact games I. Journ Math Anal Appl 40:214-225

Shapley LS (1971) Cores of convex games. International Journ of Game Theory 1:12-26

Shapley LS (1967) On balanced sets and cores. Naval Res Logist Quarterly 14:453-460 